archives-ouvertes

\title{
Experimental and numerical Lattice-Boltzmann investigation of the Darrieus-Landau instability
}

Muhammad Tayyab, Basile Radisson, Christophe Almarcha, B. Denet, Pierre Boivin

\section{- To cite this version:}

Muhammad Tayyab, Basile Radisson, Christophe Almarcha, B. Denet, Pierre Boivin. Experimental and numerical Lattice-Boltzmann investigation of the Darrieus-Landau instability. Combustion and Flame, Elsevier, 2020, 221, pp.103-109. 10.1016/j.combustflame.2020.07.030 hal-02921517

\section{HAL Id: hal-02921517 \\ https://hal.archives-ouvertes.fr/hal-02921517}

Submitted on 27 Aug 2020

HAL is a multi-disciplinary open access archive for the deposit and dissemination of scientific research documents, whether they are published or not. The documents may come from teaching and research institutions in France or abroad, or from public or private research centers.
L'archive ouverte pluridisciplinaire HAL, est destinée au dépôt et à la diffusion de documents scientifiques de niveau recherche, publiés ou non, émanant des établissements d'enseignement et de recherche français ou étrangers, des laboratoires publics ou privés. 


\title{
Experimental and numerical Lattice-Boltzmann investigation of the Darrieus-Landau instability
}

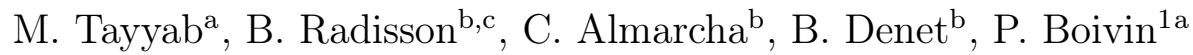 \\ ${ }^{a}$ Aix Marseille Univ, CNRS, Centrale Marseille, M2P2, Marseille, France \\ ${ }^{b}$ Aix Marseille Univ, CNRS, Centrale Marseille, IRPHE, Marseille, France \\ ${ }^{c}$ Department of Aerospace and Mechanical Engineering, University of Southern \\ California, Los Angeles, CA 90089-1191, USA
}

\begin{abstract}
We present an experimental and numerical investigation of the DarrieusLandau instability in a quasi two-dimensional Hele-Shaw cell. Experiments and Lattice-Boltzmann numerical simulations are compared with DarrieusLandau analytical theory, showing an excellent agreement for the exponential growth rate of the instability in the linear regime. The negative growth rate - second solution of the dispersion relation - was also measured numerically for the first time to the authors' knowledge. Experiments and numerical simulations were then carried out beyond the cutoff wavelength, providing good agreement even in the unexplored regime where Darrieus-Landau is supplanted by diffusive stabilization. Lastly, the non-linear evolution involving the merging of crests on the experimental flame front is also successfully recovered using both the Michelson-Sivashinsky equation integration and the Lattice-Boltzmann simulation.
\end{abstract}

Keywords: Darrieus-Landau; Lattice Boltzmann; flame instabilities; premixed flames

\footnotetext{
${ }^{1}$ Corresponding author: pierre.boivin@univ-amu.fr
} 


\section{Introduction}

Lattice-Boltzmann (LB) methods have been proven very efficient in the field of low-Mach external aerodynamics and aeroacoustics [1, 2]. However, although the first attempt of using LB methods for low Mach number combustion has been published twenty years ago [3], a lot of interest in LB methods for reactive flows has been observed only recently [4-6]. We proposed in $[6,7]$ a hybrid LB framework (Lattice-Boltzmann / finite differences) able to study combustion for low-Mach flows. Among the tests in [6] was a preliminary study of the Darrieus-Landau instability of premixed flames, a very challenging test case for LB methods since it couples temperature and species fields to the velocity field. The underlying coupling between the Lattice-Boltzmann and finite differences solvers was further improved in [8], significantly improving the method stability.

Other numerical methods have been able to compute an important characteristic of the Darrieus-Landau instability, the growth rate of small amplitude perturbations as a function of wavenumber, for one-step chemistry $[9,10]$ or for hydrogen-air flames [11], but it will be the first time that a LB method is able to describe this phenomenon. The comparison will not be limited to linear properties of the instability, but we will also show that the LB method also describes correctly the non linear evolution of the flame.

In this paper, we compare two-dimensional simulations with our hybrid LB method to quasi-2D experiments performed in a Hele-Shaw burner. Compared to [6] we will be able to obtain the full dispersion relation giving the growth rate as a function of wavenumber: it will be shown that this dis- 
persion relation has the same form as that obtained in experiments, even beyond the cutoff wave number. Furthermore, we extend the comparison up to times where non-linearities play a significant role in the flame dynamics thereby demonstrating the ability of the method to correctly predict these complex phenomena.

\section{Numerical set-up}

The numerical simulations are carried out with the PROLB software using a pressure-based hybrid regularized thermal Lattice Boltzmann (LB) model coupled with a Finite Differences (FD) solver. The probability density function $f_{i}$ (of finding gas particles at $x$ with velocity $\boldsymbol{c}_{i}$ ) is solved using hybrid regularized collision model [6]. The streaming and collision process can be expressed under discrete form as

$$
\begin{aligned}
& f_{i}(t+\delta t, \boldsymbol{x})=f_{i}^{\mathrm{col}}\left(t, \boldsymbol{x}-\boldsymbol{c}_{\boldsymbol{i}} \delta t\right), \\
& f_{i}^{\mathrm{col}}(t, \boldsymbol{x})=f_{i}^{\mathrm{eq}}+\left(1-\frac{\delta t}{\bar{\tau}}\right) f_{i}^{\mathrm{neq}}+\frac{\delta t}{2} F_{i}^{E}
\end{aligned}
$$

where $\bar{\tau}$ is a non-dimensional relaxation time, $\delta t$ is the time-step, $\boldsymbol{c}_{i}$ is the $i^{\text {th }}$ discrete velocity of the D3Q19 lattice set, and $f_{i}^{e q}$ and $f_{i}^{\text {neq }}$ the equilib-

rium and non-equilibrium segments of the distribution function and $F_{i}^{E}$ is the forcing term required to correctly recover the stress tensor [6, 12]. Full expressions for $f_{i}^{e q}, f_{i}^{n e q}, F_{i}^{E}$ and their relation with the macroscopic variables are provided in AppendixA. Note that the expressions correspond to those presented in [6], with the addition of the recent developments presented in $[8]$.

It can be shown via the Chapman-Enskog expansion $[6,8]$ that the above system of equations is equivalent to solving the mass and momentum con- 
servation equations

$$
\begin{aligned}
\frac{\partial \rho}{\partial t}+\frac{\partial}{\partial x_{\alpha}}\left(\rho u_{\alpha}\right) & =0 \\
\frac{\partial \rho u_{\alpha}}{\partial t}+\frac{\partial}{\partial x_{\beta}}\left(\rho u_{\alpha} u_{\beta}\right) & =-\frac{\partial p}{\partial x_{\alpha}}+\frac{\partial \mathcal{T}_{\alpha \beta}}{\partial x_{\beta}},
\end{aligned}
$$

at second-order in time and space, where notations follow [13].

The FD solver consists of mass conservation of species $k$ and enthalpy conservation equations solved through classical central difference approach. Temporal integration of the FD equations is explicit first-order in time, but the global order of the method is close to second-order [6, 8, 12]. Further details on the coupling between the two solvers are available in our recent publications $[6-8,12]$. The species conservation equation reads

$$
\rho \frac{\partial Y_{k}}{\partial t}+\rho u_{\alpha} \frac{\partial Y_{k}}{\partial x_{\alpha}}=\frac{\partial}{\partial x_{\alpha}}\left(-\rho Y_{k} V_{k, \alpha}\right)+\dot{\omega}_{k}
$$

where $\dot{\omega}_{k}$ is the net chemical production rate of species $k$, and $V_{k, \alpha}$ is its diffusion velocity [13]. The energy conservation can be expressed in different ways, in this work we decide to consider the enthalpy conservation of a gas mixture with multi-component ideal gas thermodynamic closure :

$$
h=\sum_{k=1}^{N} h_{k} Y_{k}, h_{k}=\int_{T_{0}}^{T} C_{p, k}(T) d T+\Delta h_{f, k}^{0},
$$

where $T$ and $h$ are linked through NASA polynomials leading to the following enthalpy equation

$$
\rho \frac{\partial h}{\partial t}+\rho u_{\alpha} \frac{\partial h}{\partial x_{\alpha}}=\frac{D p}{D t}-\frac{\partial q_{\alpha}}{\partial x_{\alpha}} .
$$

where $\frac{D p}{D t}=\frac{\partial p}{\partial t}+u_{\alpha} \frac{\partial p}{\partial x_{\alpha}}$ is neglected. The heat flux $q_{\alpha}$ reads

$$
q_{\alpha}=-\lambda \frac{\partial T}{\partial x_{\alpha}}+\rho \sum_{k=1}^{N} h_{k} Y_{k} V_{k, \alpha}
$$


with $\lambda$ the thermal conductivity. Diffusion velocities are defined as in [6], using constant Schmidt number for each species (See Table B.1), and a correction velocity to ensure mass conservation [13].

\section{Experimental set-up}

The experiments are carried out in a Hele-Shaw burner [14-20]. The apparatus used here is made of two borosilicate glass plates $(1500 \times 500 \times 5 \mathrm{~mm})$ vertically oriented and separated by a $\Delta=5 \mathrm{~mm}$ gap, shown in Fig. 1 . The burner is opened at the top, and closed at the bottom and on the two vertical sides. At the bottom of the facility a flow line controlled by two Bronkhorst EL-Flow series mass-flow regulators allows to fill the burner with a propaneair mixture with desired equivalence ratio $\varphi$. For all the experiments presented hereafter, the Hele-shaw burner is filled with a propane-air mixture $\varphi=0.8$ with a flow rate such that the mixture velocity at the top of the burner is in excess to the flame speed, allowing the flame to remain anchored as a Bunsen flame at the top of the two glass plates. Then the mixture flow is stopped (in a repeatable manner thanks to a solenoid valve) and the flame starts its downwards propagation. The initial flat front is rapidly destabilized due to Darrieus-Landau effect. Initial perturbations are either magnified or damped according to their respective growth rate, leading to typical Darrieus-Landau wrinkled flame fronts. In order to measure the growth rate associated with perturbation of different wavelengths we make use of a forcing method to select the desired wavelength. For this purpose, a steel plate, laser-cut with a sinusoidal profile, is positioned at top of the burner next to the anchored flame (see [21] for details about the experimental technique). This technique allows to print an initial perturbation on the flame front. The 


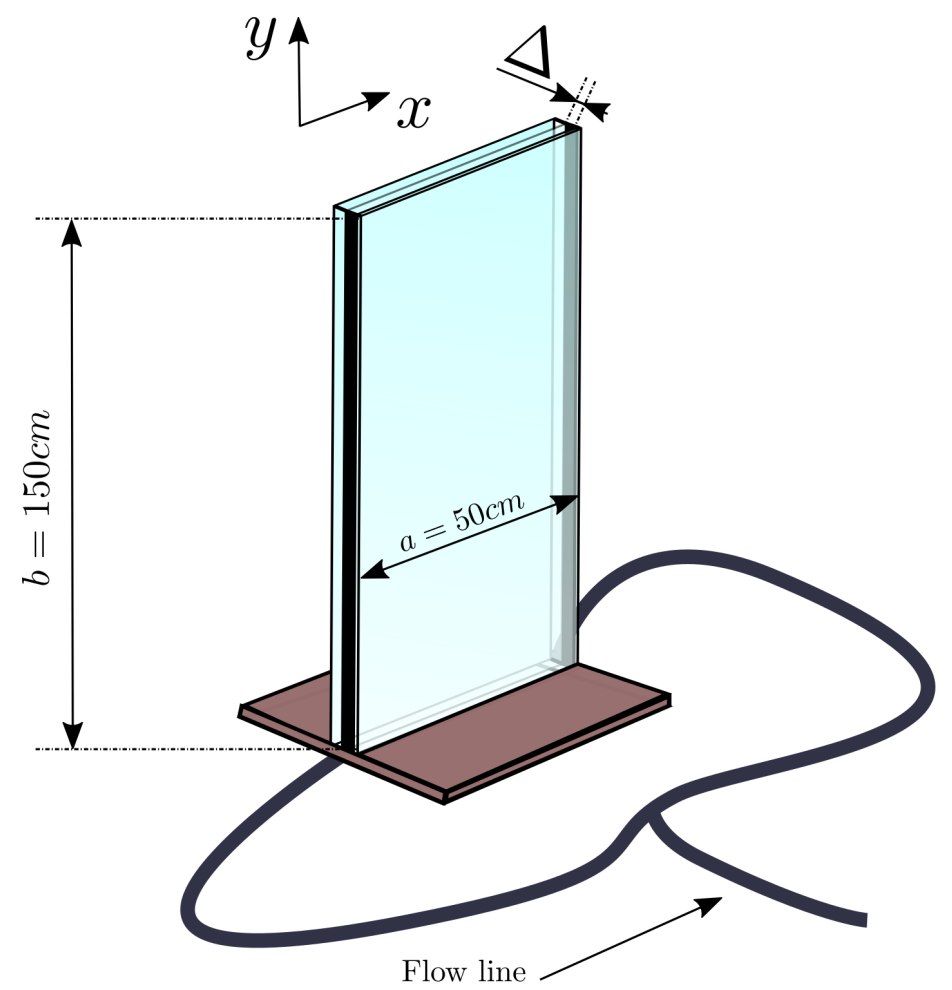

Figure 1: Experimental Set-up

flame front dynamics is then recorded using a high speed camera (Photron FASTCAM Mini AX200) and analyzed using an image processing python code (based on the scikit-image opensource library [22]).

\section{Comparison and validation}

\subsection{Numerical setup}

The equations solved with the Lattice-Boltzmann method correspond to two dimensional flames interacting with the velocity field and will be compared to quasi-2D experiments in the Hele-Shaw burner. However these equations do not correspond to the narrow-channel approximation [16] as the $5 \mathrm{~mm}$ gap used in the experiments is not sufficiently small. 
Propane-air thermochemical properties are as follow:

- NASA polynomial coefficients for the thermodynamic closure $(p, T)=$ $f(\rho, h)$ required in closing the governing equations $(2,3$ and 5$)$,

- Power-law for the viscosity, yielding transport properties via constant Prandtl and component-specific Schmidt numbers,

- One-step chemistry, where the pre-exponential parameter was chosen so the flame velocity and thickness would be in agreement with the dispersion relation. In particular, this leads to a laminar flame velocity lower than usual, to account for heat losses.

A detailed account on these thermo-chemical parameters is available in AppendixB.

In the following, we consider a two-dimensional rectangular computational domain uniformly discretized with $\delta_{x}=10^{-4} \mathrm{~m}$. The domain width corresponds exactly to one wave length $\frac{2 \pi}{k}$ for the Linear regime analysis (Sec. 4.2). In the non-linear regime study Sec. 4.3 the domain width is $160 \mathrm{~mm}$, corresponding to one third of the size of the Hele-Shaw cell represented in Fig. 1: in the experiment, the same initial profile is reproduced three times in the span-wise direction to improve periodicity. The time-step is maintained at $\delta_{t}=7.217 \times 10^{-8} \mathrm{~s}$, corresponding to an acoustic CFL of 0.63 .

The right and left boundaries conditions are periodic, while a fixed wall is set at the bottom, and an open boundary conditions is considered at the top, letting the burnt gases escape.

The domain is initialized by computing first the one-dimensional premixed flame structure, e.g. $T_{1 D}(y)$ for the temperature. That profile is then 
extended to the two-dimensional domain as $T(x, y, t=0)=T_{1 D}(y-f(x))$, where $f(x)$ is the desired geometrical perturbation:

- a single sine wave for the linear regime analysis (Sec. 4.2)

- a periodic experimental solution for the non-linear dynamics analysis (Sec. 4.3)

Buoyancy forces are not taken into account in the Lattice Boltzmann numerical scheme. For downward propagating flames such as those observed experimentally in the Hele-Shaw cell, the gravity term leads to slightly lower growth rates, and does not have a large influence on the non linear evolution for short times. Interesting papers on the role of gravity on the dispersion relation are [23] for two-dimensional flames and [24] in the narrow-channel approximation.

\subsection{Linear Regime Analysis}

The linear dynamics is analyzed by perturbing the flame front using sinusoidal waves. Initially, the amplitude of the waves are small and are expected to grow because of the Darrieus-Landau instability.

Considering that the thermal diffusive properties of the mixture depends on the temperature as $\rho D_{t h} \propto T^{\beta}$ (with $\beta=0.69$ ), the theoretical dispersion relation is then given by [23] (see also [25]) :

$$
A(k) \sigma^{2}+B(k) \sigma+C(k)=0,
$$

where $\sigma$ is the growth rate of the perturbation with wavenumber $k$. $A(k)$, $B(k), C(k)$ are coefficients depending on gas expansion $E=\rho_{u} / \rho_{b}$, Markstein number $\mathcal{M}$, laminar flame speed $u_{L}$, flame thickness $d=D_{t h} / u_{L}$, and 
Prandtl number $P r$ as

$$
\begin{aligned}
& A(k)=\frac{E+1}{E}+\frac{E-1}{E} k d\left(\mathcal{M}-J \frac{E}{E-1}\right), \\
& B(k)=u_{L} k(2+2 E k d(\mathcal{M}-J)), \\
& C(k)=u_{L}^{2} k^{2}\left((E-1) \frac{k}{k_{c}}-(E-1)\right),
\end{aligned}
$$

where the cutoff wavenumber $k_{c}$ verifies $k_{c}^{-1}=d\left(E^{\beta}+\frac{3 E-1}{E-1} \mathcal{M}-\frac{2 E}{E-1} J+\right.$ $(2 \operatorname{Pr}-1) H)$. The integrals $J$ and $H$ can be found in $[23,25]$. The positive root $\sigma_{+}$(the positive growth rate, leading to an exponential amplification of the perturbations) of the quadratic equation (7) verifies approximately

$$
\sigma_{+}=a k-b k^{2}+\mathcal{O}\left(k^{3}\right)
$$

A straightforward development (see [17]) leads to

$$
a=\frac{E}{E+1}[S-1] u_{L}
$$

where $S=\sqrt{1+E-\frac{1}{E}}$, the relation $\sigma=a k$ is the known Darrieus-Landau result without Markstein number effect, and

$$
\begin{gathered}
b=-d u_{L}\left[\left(-a^{2} \frac{E-1}{E}-2 a E\right) \mathcal{M}\right. \\
\left.+J\left(a^{2}+2 a E\right)-\frac{E-1}{k_{c} d}\right] / 2 S .
\end{gathered}
$$

This dispersion relation has also been obtained by Matalon and coworkers for temperature dependent diffusivities (see [26, 27]). 
It has been possible to measure experimentally the dispersion relation corresponding to Eq. (9) for 2D Bunsen flames [25] and recently some of the authors have measured the dispersion relation in a Hele-Shaw cell [17, 21]. Numerically some measurements of growth rates have been performed, first for one-step chemistry [9, 10, 28] and recently for hydrogen-air flames [11, 27, 29].

Note that a negative root $\sigma_{-}$of the quadratic equation (7) also exists, we will show later that we are able to measure this negative growth rate in the numerical simulations. Anyway, as the precision in the calculation decreases when the damping increases, we will refer to the approximate formula, only valid for low wavenumbers:

$$
\sigma_{-}=\frac{E}{E+1}[-S-1] u_{L} k+\mathcal{O}\left(k^{2}\right) .
$$

This mode attenuates fast and the positive mode associated to $\sigma_{+}$becomes dominant. This phenomenon is observed in the simulation results and is presented in Fig. 2. The initial instances of the growth are exponential and are defined in terms of both modes as:

$$
A=A_{0} e^{\sigma_{+} t}+A_{1} e^{\sigma_{-} t},
$$

where $\sigma_{+}$and $\sigma_{-}$correspond to the growth rates of the positive and negative mode respectively.

Our initial condition is a front slightly harmonically perturbed compared to the plane front, imposed following the strategy presented in Sec. 4.1. Let us note however that the velocity field could not be chosen in order to only have the growing mode of the instability, so that we have a transient. However, the precise transient does not change our estimate for the negative growth rate. 

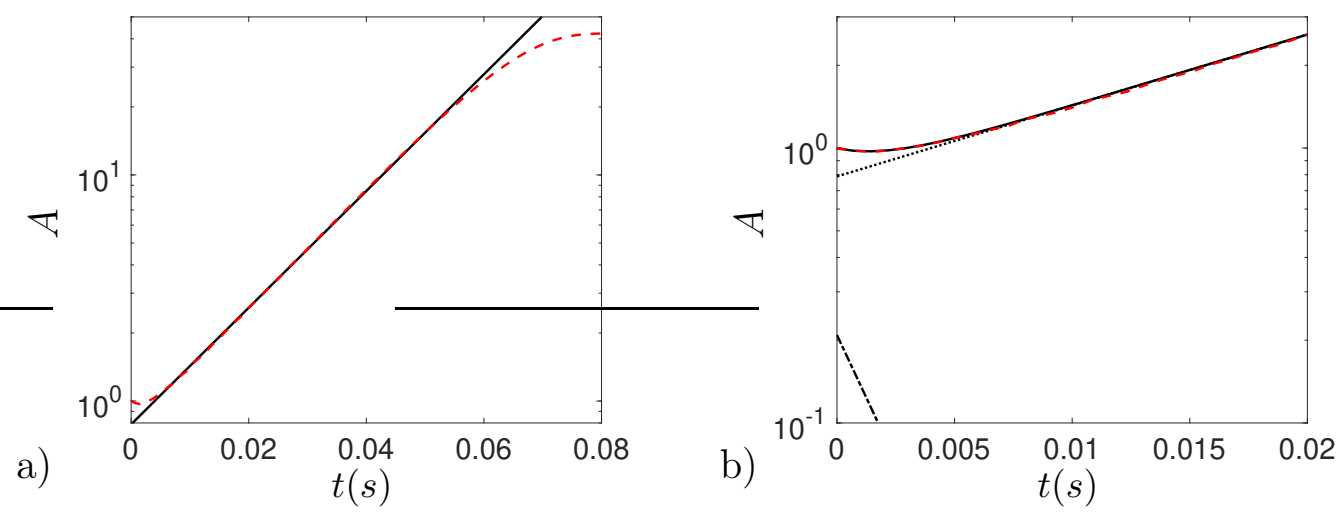

Figure 2: Linear stage dynamics. a) Evolution of normalized amplitude (A) calculated from numerical simulation (red dashed line) and gradient fit related to $\sigma_{+}$(black solid line) presented over time where development of non-linear stage is observed. b) Gradient fit related to $\sigma_{-}$(black dash-dotted line), gradient fit related to $\sigma_{+}$(black dotted line), evolution of A (red dashed line) and sum of both fits related to $\sigma_{-}$and $\sigma_{+}$(black solid line, indistinguishable from the red dashed line) presented at the initial instances where both modes $\left(\sigma_{+}, \sigma_{-}\right)$are present.

The Amplitude (A), computed using Fourier Transform, is reported in Fig. 2, for an initial sinusoidal perturbation at $k=0.52 \mathrm{~mm}^{-1}$. The fit of the positive mode $\left(\sigma_{+}\right), A=A_{0} e^{\sigma_{+} t}$ from Eq. (13) is represented in Fig. 2a. Furthermore, the initial presence of two modes is underlined in Fig. 2b, along with the fit of the negative mode $\left(\sigma_{-}\right)$. We clearly see that fitting the solution as the linear combination mentioned in Eq. (13) is accurate for all times, provided the linear regime is valid, until the amplitude of the mode saturates due to nonlinear effect.

As seen from Eq. (9), the positive mode of the growth is of the form $\sigma \propto|k|-k^{2}$. The initial growth is measured and compared to the theoretical dispersion relation, written in the following way

$$
\sigma=\frac{4 \sigma_{M}}{k_{c}}\left(|k|-\frac{k^{2}}{k_{c}}\right),
$$

where $\sigma_{M}$ is the growth rate of the most amplified wavelength and $k_{c}$ the 

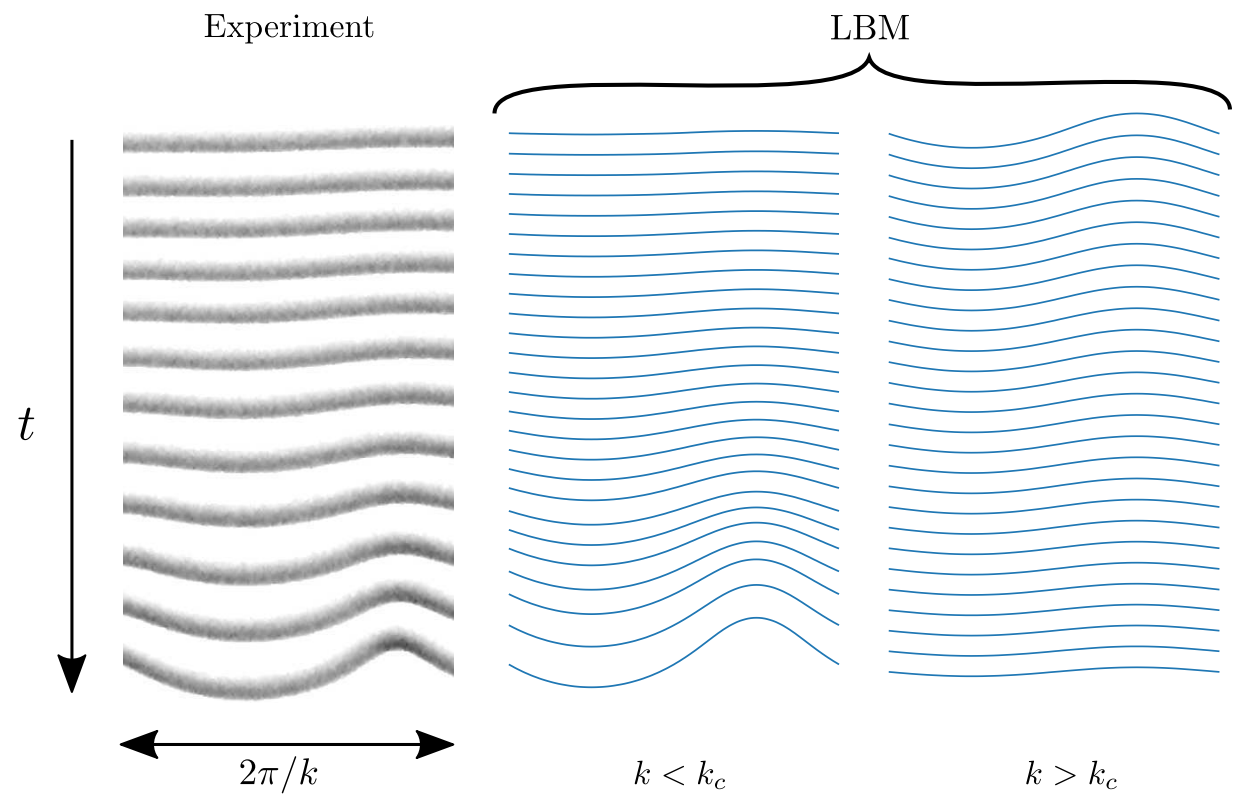

Figure 3: Observation of growth and decay with wavenumbers higher and lower than $k_{c}$. Propagation of the flame is from top to bottom. Left: Growth at wavenumber lower than $k_{c}$. Right: Decay with wavenumber higher than $k_{c}$. Experimental (Black), and Numerical (blue) results.

cutoff wavenumber. This dispersion relation is then fitted on the computed results. In order to have a quantitative agreement with the experimental results (we will only show propane-air flames with equivalence ratio $\varphi=0.8$ ), we choose in the numerical simulations the following parameters: $u_{L}=0.177$ $\mathrm{m} / \mathrm{s}, E=7.12, d=0.53 \mathrm{~mm}$. Indeed the heat losses in the Hele-Shaw burner are large and lead to a weaker hydrodynamic instability than what is observed in a tube. $u_{L}$ and $d$ have been chosen to have an agreement with the experimental dispersion relation, in particular we have taken a value for $u_{L}$ lower than the laminar flame speed for equivalence ratio $\varphi=0.8$. Further details are given in AppendixB.

Figure 3 qualitatively shows the growth of perturbations at low wavenum- 
ber and decay for a wavenumber larger than $k_{c}$. The figure in black corresponds to an experiment (propane, $\varphi=0.8$ ), and is compared to a numerical simulation for an unstable wavenumber. The figure on the right is a numerical simulation for a stable wavenumber (larger than $k_{c}$ )
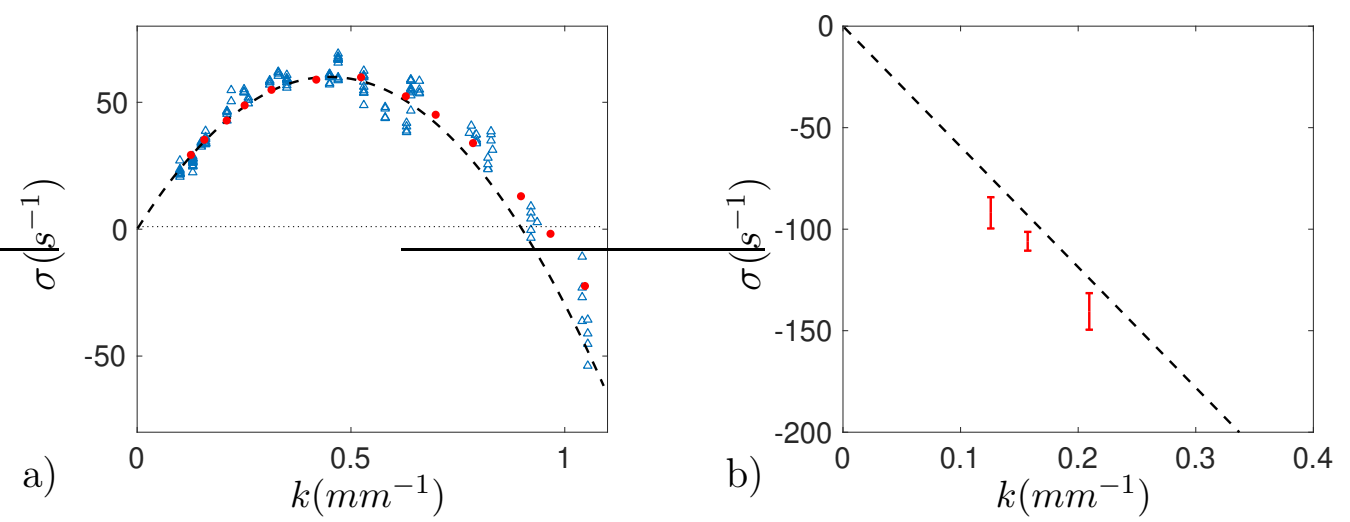

Figure 4: Quantification and comparison of $\sigma_{+}$and $\sigma_{-}$. a) Growth rate $\left(\sigma_{+}\right)$measurement obtained from the experimental (blue triangles) and numerical (red circles) investigations. The theoretical dispersion relation (black dashed line) is then fitted with $\sigma_{m}=60 \mathrm{~s}^{-1}$ and $k_{c}=0.9 \mathrm{~mm}^{-1}$. b) Growth rates $\left(\sigma_{-}\right)$computed from simulations (red error bars) and theoretical solution from Eq. (12) (black dashed line) .

Quantitative agreement between numerical and experimental results is shown in Fig. 4a, where the numerical results (red circles) are compared to experiments (blue triangles) and to a fit of the dispersion relation (Eq. 14). Note that we have also been able to measure the negative growth rate (Fig. 4b), which is compared to Eq. (12). The negative growth rates are presented as error bars, given the difficulty of measuring accurately the quantity due to initial pressure perturbations present in the computational domain. Although the Lattice-Boltzmann measurements are slightly below the theoretical curve, the agreement is relatively good. To the knowledge of the authors, this is the first time in the literature that this negative growth rate 
is measured.

We thus have evidenced that our hybrid LBM method is able to correctly describe the linear premixed flame dynamics. It will be demonstrated in the next subsection that an agreement is also possible for the non linear evolution of the flame.

\subsection{Non-Linear Dynamics}

A very unstable premixed flame (a flame in a large domain, often called a self-turbulent flame [30]) leads to a complex non linear evolution involving merging and creation of new crests on the flame front. We have to show that our numerical simulations are able to describe correctly these effects. The LB simulations will thus be compared to experiments (see also [31]) and to the results of the Sivashinsky equation (or Michelson Sivashinsky : MS equation) [32]. This equation, solved with periodic boundary conditions, includes the linear dispersion relation (Eq. 14), and a quadratic non-linearity and writes as:

$$
\phi_{t}+\frac{u_{A}}{2} \phi_{x}^{2}=\frac{4 \sigma_{M}}{k_{c}}\left(\frac{\phi_{x x}}{k_{c}}+I(\phi, x)\right),
$$

where $\phi$ stands for the vertical position of the front, $x$ is the transverse coordinate and $t$ the time. $\sigma_{M}$ and $k_{c}$ are the parameters given in Eq. (14), $k_{c}$ is the cut-off wavenumber, $I(\phi, x)$ is the Landau operator corresponding to a multiplication by $|k|$ in Fourier space. The curvature term $\phi_{x x}$ comes from the thermal diffusive restabilization and is responsible for the damping of disturbances at small scales. Finally, $\phi_{x}^{2}$ is a non linear term responsible for the formation of cusps. For low expansion $E, u_{A}$ is close to the laminar flame speed, this is no longer the case for large $E[33,34]$. This parameter 
$u_{A}$ is fitted to obtain amplitudes of the flame comparable to experiments.

In order, to compare the evolution given by the LBM integration and the MS model with an experiment, a flame is ignited at the top of the Hele-Shaw as a forced inverted V-flame. After a few instants of propagation, when the cusps start to be visible on the flame front, we extract the flame contour $(y=f(x))$ from the recorded images using an image processing algorithm. The obtained flame contour is then used as an initial condition for numerical integration $\left(\mathrm{MS}: \phi(x, t=0)=f(x) \operatorname{LBM}: T(x, y, t=0)=T_{1 D}(y-f(x))\right)$. In Fig. 5, we show the comparison between the evolution of experimental propane-air fronts $(\varphi=0.8)$, (Top figure, in black), the LB simulations, in blue, and the MS equation (Bottom figure, green lines). In each case, the positions of the cusps are highlighted, in order to better compare the three different evolutions.

As can be seen in the figure, the crest merging process is correctly described for short times by both the LB simulations and the Sivashinsky equation. The LB simulations are actually closer to experiments, which may be caused by the fact that the LB dispersion relation is slightly larger compared to the MS one close to $k_{c}$ (see Fig. 4) or by the fact that flow non-linearities (neglected at the first order in gas expansion in the MS model) play a significant role. A difference between the experiments and the other figures is the creation of new cusps. This effect, which is not observed in the LB or MS simulations, could be caused by effects that we have neglected, such as additional noise, or gravity (the experimental premixed flames are actually propagating downwards). 


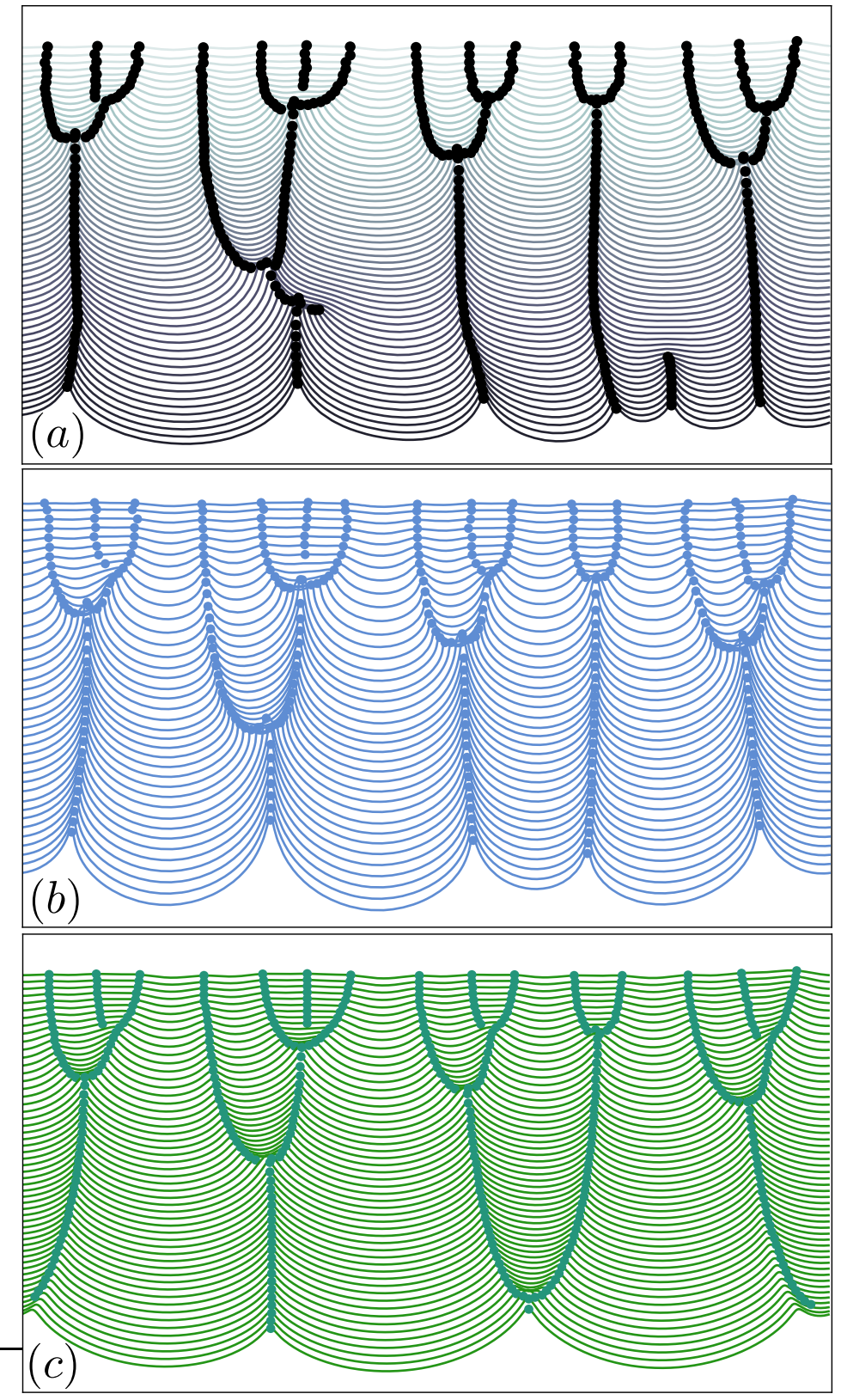

Figure 5: Darrieus-Landau non linear evolution: (a) Experimental, (b) Numerical results and (c) MS equation starting from the same initial condition extracted from the experiment. 


\section{Conclusion}

Simulations performed with an hybrid Lattice-Boltzmann model for lowMach reactive flows were presented in this paper for premixed flames unstable relative to the Darrieus-Landau instability. We were able to compare quantitatively the numerical results with quasi 2D experiments in a Hele-Shaw cell. The growth rates of the instability were measured by studying the development of small amplitude perturbations, and it was even possible to measure the decaying mode of the instability, showing the precision of the method. A correct agreement with experiments was also observed for the non linear evolution of the flame front.

\section{Acknowledgements}

Centre de Calcul Intensif d'Aix-Marseille is acknowledged for granting access to its high performance computing resources. This work was supported by the French National Research Agency under agreement ANR-14-CE050006, and received funding from Excellence Initiative of Aix-Marseille University - A*MIDEX, a French "Investissements d'Avenir" programme. It has been carried out in the framework of the Labex-MEC.

[1] S. Chen, G. D. Doolen, Lattice Boltzmann method for fluid flows, Annu. Rev. Fluid Mech. 30 (1) (1998) 329-364.

[2] A. Sengissen, J.-C. Giret, C. Coreixas, J.-F. Boussuge, Simulations of lagoon landing-gear noise using Lattice Boltzmann solver, in: 21st AIAA/CEAS Aeroacoustics Conference, 2015, p. 2993. 
[3] O. Filippova, D. Hänel, A novel lattice BGK approach for low mach number combustion, J. Comput. Phys. 158 (2) (2000) 139-160.

[4] C. Lin, K. H. Luo, Mesoscopic simulation of nonequilibrium detonation with discrete Boltzmann method, Combust. Flame 198 (2018) 356-362.

[5] S. A. Hosseini, H. Safari, N. Darabiha, D. Thévenin, M. Krafczyk, Hybrid Lattice Boltzmann-finite difference model for low mach number combustion simulation, Combust. Flame 209 (2019) 394-404.

[6] M. Tayyab, S. Zhao, Y. Feng, P. Boivin, Hybrid regularized LatticeBoltzmann modelling of premixed and non-premixed combustion processes, Combust. Flame 211 (2020) 173-184.

[7] Y. Feng, M. Tayyab, P. Boivin, A Lattice-Boltzmann model for lowmach reactive flows, Combust. Flame 196 (2018) 249 - 254.

[8] G. Farag, S. Zhao, T. Coratger, P. Boivin, G. Chiavassa, P. Sagaut, A pressure-based regularized lattice-boltzmann method for the simulation of compressible flows, Phys. Fluids accepted.

[9] B. Denet, P. Haldenwang, A numerical study of premixed flames Darrieus-Landau instability, Combust. Sci. Technol. 104 (1-3) (1995) $143-167$.

[10] S. Kadowaki, T. Hasegawa, Numerical simulation of dynamics of premixed flames: flame instability and vortex-flame interaction, Prog. Energy Combust. Sci. 31 (3) (2005) 193-241. 
[11] C. E. Frouzakis, N. Fogla, A. G. Tomboulides, C. Altantzis, M. Matalon, Numerical study of unstable hydrogen/air flames: Shape and propagation speed, Proc. Combust. Inst. 35 (2015) 1087-1095.

[12] Y. Feng, P. Boivin, J. Jacob, P. Sagaut, Hybrid recursive regularized thermal lattice boltzmann model for high subsonic compressible flows, J. Comput. Phys. 394 (2019) $82-99$.

[13] T. Poinsot, D. Veynante, Theoretical and numerical combustion, RT Edwards, Inc., 2005.

[14] G. Joulin, G. Sivashinsky, Influence of momentum and heat losses on the large-scale stability of quasi-2D premixed flames, Combust. Sci. Technol. $98(1-3)(1994)$ 11-23.

[15] J. Sharif, M. Abid, P. D. Ronney, Premixed-gas flame propagation in Hele-Shaw cells, in: 1st Joint U.S. Sections Combustion Institute Meeting, Washington, DC; United States, 1999.

[16] D. Fernández-Galisteo, V. N. Kurdyumov, P. D. Ronney, Analysis of premixed flame propagation between two closely-spaced parallel plates, Combust. Flame 190 (2018) 133-145.

[17] E. Al Sarraf, C. Almarcha, J. Quinard, B. Radisson, B. Denet, P. GarciaYbarra, Darrieus-Landau instability and Markstein numbers of premixed flames in a hele-shaw cell, Proc. Combust. Inst. 37 (2) (2019) 1783-1789.

[18] H. J. Jang, G. M. Jang, N. I. Kim, Unsteady propagation of premixed 
methane/propane flames in a mesoscale disk burner of variable-gaps, Proc. Combust. Inst. 37 (2) (2019) 1861-1868.

[19] M. M. Alexeev, O. Y. Semenov, S. E. Yakush, Experimental Study on Cellular Premixed Propane Flames in a Narrow Gap between Parallel Plates, Combust. Sci. Technol. (2018) 1-20.

[20] F. Veiga-López, D. Martínez-Ruiz, E. Fernández-Tarrazo, M. SánchezSanz, Experimental analysis of oscillatory premixed flames in a HeleShaw cell propagating towards a closed end, Combust. Flame 201 (2019) $1-11$.

[21] E. Al Sarraf, C. Almarcha, J. Quinard, B. Radisson, B. Denet, Quantitative Analysis of Flame Instabilities in a Hele-Shaw Burner, Flow, Turbulence and Combustion 101 (3) (2018) 851-868.

[22] S. van der Walt, J. L. Schönberger, J. Nunez-Iglesias, F. Boulogne, J. D. Warner, N. Yager, E. Gouillart, T. Yu, the scikit-image contributors, scikit-image: image processing in Python, PeerJ 2 (2014) e453.

[23] P. Clavin, P. Garcia, The influence of the temperature dependence of diffusivities on the dynamics, Journal de Mécanique Théorique et Appliquée 2 (2) (1983) 245-263.

[24] D. Fernández-Galisteo, V. N. Kurdyumov, Impact of the gravity field on stability of premixed flames propagating between two closely spaced parallel plates, Proc. Combust. Inst. 37 (2) (2019) 1937-1943.

[25] J.-M. Truffaut, G. Searby, Experimental study of the Darrieus-Landau 
instability on an inverted- $\mathrm{V}$ flame, and measurement of the Markstein number, Combust. Sci. Technol. 149 (1-6) (1999) 35-52.

[26] M. Matalon, C. Cui, J. K. Bechtold, Hydrodynamic theory of premixed flames: effects of stoichiometry, variable transport coefficients and arbitrary reaction orders, J. Fluid Mech. 487 (2003) 179-210.

[27] C. Altantzis, C. E. Frouzakis, A. G. Tomboulides, M. Matalon, K. Boulouchos, Hydrodynamic and thermodiffusive instability effects on the evolution of laminar planar lean premixed hydrogen flames, J. Fluid Mech. 700 (2012) 329-361.

[28] P. E. Lapenna, R. Lamioni, G. Troiani, F. Creta, Large scale effects in weakly turbulent premixed flames, Proc. Combust. Inst. 37 (2) (2019) 1945-1952.

[29] L. Berger, K. Kleinheinz, A. Attili, H. Pitsch, Characteristic patterns of thermodiffusively unstable premixed lean hydrogen flames, Proc. Combust. Inst. 37 (2) (2019) 1879-1886.

[30] C. Almarcha, J. Quinard, B. Denet, E. Al-Sarraf, J. Laugier, E. Villermaux, Experimental two dimensional cellular flames, Phys. Fluids 27 (2015) 091110.

[31] C. Almarcha, B. Radisson, E. Al Sarraf, E. Villermaux, B. Denet, J. Quinard, Interface dynamics, pole trajectories, and cell size statistics, Physical Review E 98 (3) (2018) 030202. 
[32] G. Sivashinsky, Nonlinear analysis of hydrodynamic instability in laminar flames - i. derivation of basic equations, Acta Astronaut. 4 (1977) $1177-1206$.

[33] G. Sivashinsky, P. Clavin, On the nonlinear theory of hydrodynamic instability in flames, J. Phys. 48 (2) (1987) 193-198.

[34] K. A. Kazakov, On-shell description of stationary flames, Phys. Fluids 17 (3) (2005) 032107.

[35] O. Colin, F. Ducros, D. Veynante, T. Poinsot, A thickened flame model for large eddy simulations of turbulent premixed combustion, Phys. Fluids 12 (2000) 1843.

\section{AppendixA. Expressions for the LBM solver}

In the low-Mach number approximation, the equilibrium function can be truncated at second-order as

$$
\begin{aligned}
& f_{i}^{\mathrm{eq}}=w_{i}\left[\rho \theta+\frac{\rho c_{i \alpha} u_{\alpha}}{c_{s}^{2}}+\frac{\mathcal{H}_{i, \alpha \beta}^{(2)} a_{\alpha \beta}^{(2), \mathrm{eq}}}{2 c_{s}^{4}}\right], \\
& f_{i}^{\text {neq }}=\frac{\omega_{i}}{2 c_{s}^{4}} \mathcal{H}_{i, \alpha \beta}^{(2)} a_{\alpha \beta}^{\text {neq }},
\end{aligned}
$$

where,

$$
\begin{aligned}
& \mathcal{H}_{i, \alpha \beta}^{(2)}=c_{i \alpha} c_{i \beta}-c_{s}^{2} \delta_{\alpha \beta}, \quad a_{\alpha \beta}^{(2) \text {,eq }}=\rho u_{\alpha} u_{\beta}, \\
& a_{\alpha \beta}^{\text {neq }}=\mathcal{H}_{i, \alpha \beta}^{(2)}\left(f_{i}-f_{i}^{\text {eq }}\right),
\end{aligned}
$$

$\theta$ is the normalized temperature linked with pressure as $p=\rho c_{s}^{2} \theta$, using ideal gas law, $w_{i}$ is the weight coefficient related to the discrete velocity $\boldsymbol{c}_{i}$ and $c_{s}$ is the lattice sound speed. Note that the equilibrium function differs from 
[6], and was adapted from [8], enhancing numerical stability. In particular, the pressure contribution $\theta$ is now inserted in the zero-th moment of (A.2) rather than higher moments as in [6].

Consequently [8], the reconstruction of macroscopic variables now read

$$
\begin{aligned}
& \rho(t+\delta t, \boldsymbol{x})=\sum f_{i}^{\mathrm{col}}+\rho(t, \boldsymbol{x})(1-\theta(t, \boldsymbol{x})) \\
& \left(\rho u_{\alpha}\right)(t+\delta t, \boldsymbol{x})=\sum c_{i \alpha} f_{i}^{\mathrm{col}} .
\end{aligned}
$$

Finally, the required forcing term is obtained as

$$
\begin{aligned}
F_{i}^{E}= & \frac{\omega_{i}}{2 c_{s}^{4}} \mathcal{H}_{i, \alpha \beta}^{(2)}\left[c_{s}^{2} u_{\alpha} \rho_{, \beta}+c_{s}^{2} u_{\beta} \rho_{, \alpha}+\delta_{\alpha \beta} \rho c_{s}^{2}\left(\frac{2}{3}-\frac{\eta_{B}}{\mu}\right) u_{\gamma, \gamma}\right. \\
& \left.+\Delta\left(\rho u_{\alpha} u_{\beta}\right)-\delta_{\alpha \beta} c_{s}^{2} \Delta[\rho(1-\theta)]\right],
\end{aligned}
$$

with $\eta_{B}$ is the bulk viscosity, and

$$
\begin{aligned}
& \Delta\left(\rho u_{\alpha} u_{\beta}\right)=\left(\rho u_{\alpha} u_{\beta}\right)(t+\delta t, \boldsymbol{x})-\left(\rho u_{\alpha} u_{\beta}\right)(t, \boldsymbol{x}), \\
& \Delta[\rho(1-\theta)]=\rho(t+\delta t, \boldsymbol{x})(1-\theta(t+\delta t, \boldsymbol{x}))-\rho(t, \boldsymbol{x})(1-\theta(t, \boldsymbol{x})) .
\end{aligned}
$$

\section{AppendixB. Numerical simulation parameters}

In this article we consider one-step propane/air mixture with 5 species: $\mathrm{C}_{3} \mathrm{H}_{8}, \mathrm{O}_{2}, \mathrm{H}_{2} \mathrm{O}, \mathrm{CO}_{2}, \mathrm{~N}_{2}$. The thermal and species diffusion coefficients are calculated using Schmidt and Prandtl numbers, detailed in Table B.1. Along with the use of viscosity's power law,

$$
\mu=\mu_{\oslash}\left(\frac{T}{T_{\oslash}}\right)^{\beta},
$$


Table B.1: Prandtl number and Schmidt numbers for each specie.

$\begin{array}{cc}P r & 0.682 \\ S_{\mathrm{C}_{3} \mathrm{H}_{8}} & 1.241 \\ S_{\mathrm{O}_{2}} & 0.728 \\ S_{\mathrm{CO}_{2}} & 0.941 \\ S_{\mathrm{H}_{2} \mathrm{O}} & 0.537 \\ S_{\mathrm{N}_{2}} & 0.690\end{array}$

where reference viscosity $\mu_{\oslash}=1.782 \times 10^{-5}$, reference Temperature $T_{\oslash}=300$ $\mathrm{K}$ and coefficient $\beta=0.69$. Furthermore, classical NASA polynomials for each species $k$ are used to define the thermodynamic properties.

In order to define the chemical source term a one-step Arrhenius kinetic model is used, following global reaction $\mathrm{C}_{3} \mathrm{H}_{8}+5 \mathrm{O}_{2} \longrightarrow 3 \mathrm{CO}_{2}+4 \mathrm{H}_{2} \mathrm{O}$, associated with the kinetic rate $\omega=k_{\text {chem }} \cdot C_{\mathrm{C}_{3} \mathrm{H}_{8}} \cdot C_{\mathrm{O}_{2}} \cdot e^{-E_{a} / R T}$, where $k_{\text {chem }}=$ $8.77 \times 10^{13} \mathrm{~cm}^{3} \mathrm{~mol}^{-1} \mathrm{~s}^{-1}, E_{a}=30 \mathrm{kcal} / \mathrm{mol}$, and $C_{i}$ are the molar concentrations for species $i$. The initial conditions used in order to compute 1-D premixed profiles at equivalence ratio $(\phi=0.8)$ are detailed in Table B.2.

Table B.2: Initial conditions: 1-D domain is initialized with fresh gases corresponding to $(0: L / 2)$ and burnt gases $(L / 2: L)$

\begin{tabular}{lll} 
Variables & fresh gases & burnt gases \\
\hline $\mathrm{T}$ & $300 \mathrm{~K}$ & $2069.98 \mathrm{~K}$ \\
$\mathrm{p}$ & $1 \mathrm{~atm}$ & $1 \mathrm{~atm}$ \\
$Y_{\mathrm{C}_{3} \mathrm{H}_{8}}$ & $4.88 \times 10^{-2}$ & $6.76 \times 10^{-15}$ \\
$Y_{\mathrm{O}_{2}}$ & $2.21 \times 10^{-1}$ & $4.39 \times 10^{-2}$ \\
$Y_{\mathrm{CO}_{2}}$ & $9.74 \times 10^{-18}$ & $1.46 \times 10^{-1}$ \\
$Y_{\mathrm{H}_{2} \mathrm{O}}$ & $1.54 \times 10^{-13}$ & $8.02 \times 10^{-2}$ \\
$Y_{\mathrm{N}_{2}}$ & $7.29 \times 10^{-1}$ & $7.29 \times 10^{-1}$
\end{tabular}

Given that the model presented in Sec. 2 does not account for heat losses, experimental results can not be compared to the simulated result. 
In order to overcome this problem, we used Eq. (10) and relation $\sigma=a k$ to compute the flame speed $S_{L}^{e x p}=0.177 \mathrm{~m} / \mathrm{s}$ which corresponds to growth obtained experimentally at lower wave-numbers. Furthermore, the original value $k_{\text {chem }}=9.9 \times 10^{13} \mathrm{~cm}^{3} \mathrm{~mol}^{-1} \mathrm{~s}^{-1}[7]$ associated to the chemical reaction rate is modified to $k_{\text {chem }}=8.77 \times 10^{13} \mathrm{~cm}^{3} \mathrm{~mol}^{-1} \mathrm{~s}^{-1}$ in order to recover $S_{L}^{e x p}$ in our simulations. Lastly, to assure that we recover comparable $\sigma_{m}$, flame thickening model [35] with a factor of 1.48 is used to recover the flame thickness. These adjustments allow us to compare the growth rates with the experimental results. 